\title{
Combinatorial Topology Of Multipartite Entangled States
}

\author{
Roman R. Zapatrin \\ Quantum Information Group, ISI, Villa Gualino, \\ Viale Settimio Severo 65, 10133, Torino, Italy; \\ e-mail: zapatrin@isiosf.isi.it
}

February 9, 2020

\begin{abstract}
With any state of a multipartite quantum system its separability polytope is associated. This is an algebro-topological object (nontrivial only for mixed states) which captures the localisation of entanglement of the state. Particular examples of separabilty polytopes for 3-partite systems are explicitly provided. It turns out that this characterisation of entanglement is associated with simulation of arbitrary unitary operations by 1- and 2-qubit gates, namely, I provide the topological description of how entanglement changes in course of such simulation.
\end{abstract}

\section{Introduction}

Entanglement in multipartite quantum system is now treated as a key resource in quantum information processing. That is why multiple efforts are drawn to quantification of entanglement for quantum states.

For bipartite quantum systems all entanglement measures are necessarily of numerical nature. In the case of multipartite systems the situation differs drastically. It was observed that even pure states of a 3-particle system can be entangled in different ways, which can not be interconverted by local unitary transformations. 
In this paper, starting from the classification of mixed bipartite states [8] I introduce a non-numerical, topological characterisation of mixed $\mathrm{N}$ partite quantum states. It is known that, in contrast with the classical case, a quantum state can be represented by different inequivalent ensembles of pure states. I consider equivalence classes of ensembles with respect to their localisation. With each state a polytope (a geometrical object in an affine space) is associated such that every maximal simplex of the polytope represents a class of equivalently localised ensembles.

To make the paper complete, all the necessary definitions are provided.

\section{Composite systems and partitions}

Let $\mathfrak{S}$ be a $N$-partite physical system. We may dwell on a less detailed description of multipartite nature of $\mathfrak{S}$, namely, grouping subsystems in their most detailed setting and considering these groups as subsystems. Each such passage to a less detailed description of the composite structure of $\mathfrak{S}$ we associate with appropriate partition of the total set $\mathfrak{A}$ of $N$ subsystems of $\mathfrak{S}$.

Denote the collection of all partitions of $\mathfrak{A}$ by $\Pi(\mathfrak{A})$ or $\Pi(N)$ where $N$ is the number of the subsystems. The first observation is that partitions are ordered.

A partition $\Sigma$ is called FINER than a partition $\Sigma^{\prime}$ (and $\Sigma^{\prime}$ is, respectively, COARSER than $\Sigma$ whenever any element of $\Sigma^{\prime}$ is a union of elements of the partition $\Sigma$. Denote it

$$
\Sigma^{\prime} \preceq \Sigma
$$

The order $\preceq$ on partitions is partial, that is, two partitions may be incomparable. To elucidate it, consider an example.

Example. Let $\mathfrak{S}$ be a 4-partite system, enumerate its components by num-

bers $\mathfrak{A}=\{1,2,3,4\}$. Consider the following partitions $\Sigma=\{12,3,4\}, \Sigma^{\prime}=$ $\{12,34\}, \Sigma^{\prime \prime}=\{13,24\}$ : 


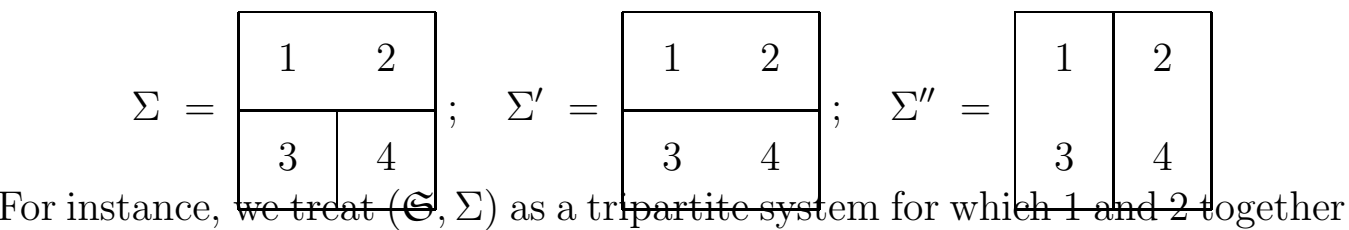
are treated as one subsystem while $\left(\mathfrak{S}, \Sigma^{\prime}\right)$ is bipartite. Among the partitions presented above

$$
\Sigma^{\prime} \preceq \Sigma
$$

while $\Sigma^{\prime \prime}$ is not comparable with any of $\Sigma, \Sigma^{\prime}$.

To summarize, we observe that besides the initial, finest decomposition of the state space $\mathcal{H}$ of the composite system $\mathfrak{S}$ we may consider coarser decompositions each of which associated with certain partition of the set $\mathfrak{A}$ of subsystems of $\mathfrak{S}$. Let us denote it as follows. The initial decomposition of $\mathcal{H}$ is

$$
\mathcal{H}=\otimes_{i \in \mathfrak{A}} \mathcal{H}_{i}
$$

and each partition $\Sigma=\left\{\sigma_{1}, \ldots \sigma_{M}\right\}$ (where $\sigma_{1} \cup \cdots \cup \sigma_{M}=\mathfrak{A}$ ) gives rise to the following decomposition of the overall state space $\mathcal{H}$ as:

$$
\mathcal{H}=\mathcal{H}_{\sigma_{1}} \otimes \cdots \otimes \mathcal{H}_{\sigma_{M}}
$$

\subsection{Decomposability, separability and entanglement}

Given a state of a composite $N$-partite system $\mathfrak{S}$, denote its density matrix by $\rho$, and first introduce the notion of decomposability.

A density matrix $\rho$ is called DECOMPOSABLE if it can be represented as a tensor product of density matrices of subsystems

$$
\rho=\rho_{1} \otimes \ldots \otimes \rho_{N}
$$


A state $\rho$ is SEPARABLE if its density matrix is a convex combination of decomposable ones.

$$
\rho=\sum p_{\alpha} \rho_{1}^{\alpha} \otimes \ldots \otimes \rho_{N}^{\alpha}
$$

with $p_{\alpha} \geq 0$ and $\sum p_{\alpha}=1$.

The states which are not separable are called ENTANGLED. It worth mentioning that for classical systems any state is separable, therefore the notion of entangled states makes sense only for quantum systems.

\subsection{The relativity of multipartite entanglement}

Now let us weaken the condition for states of a composite system $\mathfrak{S}$ to be decomposable and separable. Namely, instead of requiring (3) for the tensor product

$$
\mathcal{H}=\mathcal{H}_{1} \otimes \cdots \otimes \mathcal{H}_{N}
$$

we make this condition relative with respect to a partition $\Sigma$ of the set $\mathfrak{A}$ of subsystems of $\mathfrak{S}$, that is, with respect to the decomposition (2).

Given a partition $\Sigma=\left\{\sigma_{1}, \ldots, \sigma_{M}\right\}$ of $\mathfrak{A}$ and a a density matrix $\rho$ in the state space of $\mathfrak{S}, \rho$ is called $\Sigma$-DECOMPOSABLE whenever it can be represented as a tensor product

$$
\rho=\rho_{\sigma_{1}} \otimes \cdots \otimes \rho_{\sigma_{M}}
$$

and $\Sigma$-SEPARABLE if it is a convex combination of $\Sigma$-decomposable states:

$$
\rho=\sum p_{\alpha} \rho_{\sigma_{1}}^{\alpha} \otimes \ldots \otimes \rho_{\sigma_{N}}^{\alpha}
$$


In other words, (5) means that we can prepare $\rho$ as an ensemble of mixed states located at sites $\sigma_{1}, \ldots, \sigma_{M}$.

Given a state $\rho$, we may now ask for each partition $\Sigma$ of the set $\mathfrak{A}$ of subsystems of $\mathfrak{S}$ if $\rho$ is $\Sigma$-separable or not. As a result we obtain the set $\Pi(\rho)$ of partitions of $\mathfrak{A}$ with respect to which $\rho$ is separable (5):

$$
\Sigma \in \Pi(\rho) \quad \Leftrightarrow \quad \rho \quad \text { is } \Sigma \text {-separable }
$$

For a given state $\rho$ the set $\Pi(\rho)$ has the following property [8]:

$$
\forall \rho \quad\left\{\begin{array}{l}
\Sigma \in \Pi(\rho) \\
\Sigma^{\prime} \preceq \Sigma
\end{array} \quad \Leftrightarrow \quad \Sigma^{\prime} \in \Pi(\rho)\right.
$$

Corollary. To specify $\Pi(\rho)$ we need to provide only maximal (with respect to the order (11), that is, finest) partitions $\Sigma$ such that $\rho$ is $\Sigma$-separable. This provides us a natural geometrical picture which will be coined partition polytopes. For the sake of self-consistency, I first recall the necessary definitions.

\subsection{Simplices and polytopes}

Suppose we have a real affine space $\mathbf{A}$ of sufficiently high dimension, and there are $M+1$ independent points there. The convex hull of these points is called SIMPLEX of dimension $M$. For example:

A cut $\longrightarrow$ is a 1-simplex.

A triangle

A tetrahedron

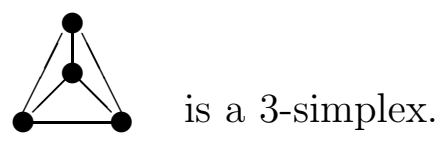

and so on. The points we have connected are called VERTICES of the simplex. The convex hulls of $K+1$ vertices in a simplex are called its FACES. In particular, 0-faces are vertices, 1 -faces are called edges. 
A polytope is a collection $\left\{\mathcal{S}^{i}\right\}$ of simplices in the space $\mathbf{A}$ having the following property

$$
\forall i, i^{\prime} \quad \mathcal{S}^{i} \cap \mathcal{S}^{i^{\prime}}=\left\{\begin{array}{l}
\text { a face of both } \mathcal{S}^{i}, \mathcal{S}^{i^{\prime}} \\
\emptyset
\end{array}\right.
$$

The VERTICES of a polytope is the set of all vertices of all its simplices, denote it by $V$

$$
V=\cup_{i} V\left(\mathcal{S}^{i}\right)
$$

For instance
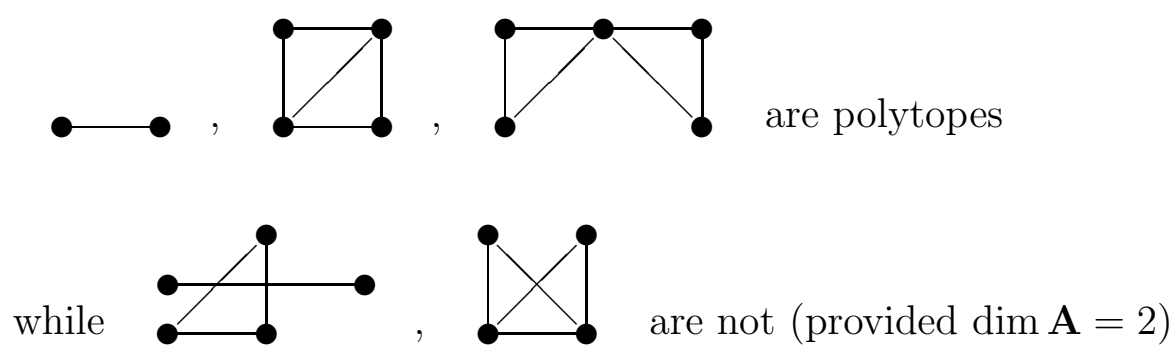

Let $\mathcal{S}, \mathcal{S}^{\prime}$ be two simplices in affine spaces $\mathbf{A}, \mathbf{A}^{\prime}$.

An affine mapping $\phi: \mathbf{A} \rightarrow \mathbf{A}^{\prime}$ is called SIMPLICIAL whenever the following conditions hold:

- Any vertex $v$ of $\mathcal{S}$ is mapped onto a vertex of $\mathcal{S}^{\prime}$. This mapping is not necessarily injective, different vertices may be mapped to one.

- 'Simplicial mappings do not brake skeletons': if $\left\{v_{1}, \ldots, v_{K}\right\}$ is a face of $\mathcal{S}$ then its image is a face as well (of perhaps smaller dimensionality).

which can be symbolically written down as:

$$
\phi(\mathcal{S}) \subseteq \mathcal{S}^{\prime}
$$




\section{Topological expression of physical proper- ties}

Now we have in our disposal all the necessary mathematics to express certain physical properties of a state $\rho$ in a topological fashion. First I introduce the appropriate objects associated with states, namely, the separability polytopes and then provide some examples, both trivial and non-trivial.

\subsection{Separability polytopes}

Now let us show how the introduced geometrical objects can represent relations between different partitions. With any partition $\Sigma_{\alpha}$ of the set $\mathfrak{A}$ we associate a simplex whose vertices are in 1-1 correspondence with the elements of the partition $\Sigma(=$ disjoint subsets of $\mathfrak{A})$.

$$
\mathcal{S}_{\alpha}=\mathcal{S}\left(\Sigma_{\alpha}\right)
$$

The separability polytope of a state $\rho$ of $\mathfrak{S}$ is built as follows:

(i) Take all maximal partitions $\left\{\Sigma^{1}, \ldots, \Sigma^{K}\right\}$ with respect to which $\rho$ is separable.

(ii) Consider the set $V$ whose elements are all elements of the partitions $\left\{\Sigma^{1}, \ldots, \Sigma^{K}\right\}$ (that is, subsets of $\mathfrak{A}$ ). Formally it is just:

$$
V=\left\{\Sigma^{1} \cup \cdots \cup \Sigma^{K}\right\}
$$

and denote by $D$ the cardinality of the set $V$.

(iii) Take an affine space $\mathbf{A}$ of sufficiently high dimension and place there $D$ independent points corresponding to the elements of $V$. They will be the vertices of the future separability polytope.

(iv) For each partition $\Sigma^{i}$ form the convex hull of appropriate vertices. 
The result we call the SEPARABILITY POLYTOPE of the state $\rho$, denote it $\mathcal{K}(\rho)$ :

$$
\mathcal{K}=\cup\left\{\mathcal{S}\left(\Sigma^{i}\right) \mid \Sigma^{i} \in \Pi(\rho)\right\}
$$

Yet separability polytopes remain an abstract geometrical object for which physical interpretation is to be provided. Recall that when we are dealing with quantum communications, a quantum state in general does not contain complete information about an ensemble which represents this state (again, this is not the case for classical systems where the correspondence stateensemble is unique).

What tells us the fact that separability of a state $\rho$ Is separable with respect to a partition $\Sigma=\left\{\sigma_{1}, \ldots, \sigma_{M}\right\}$ ? This means that the state $\rho$ can be represented as a mixture of pure states of the form:

$$
\rho=\sum p^{\alpha}\left|\Psi_{\sigma_{1}}^{\alpha}\right\rangle\left\langle\Psi_{\sigma_{1}}^{\alpha}|\otimes \cdots \otimes| \Psi_{\sigma_{M}}^{\alpha}\right\rangle\left\langle\Psi_{\sigma_{M}}^{\alpha}\right|
$$

If the partition $\Sigma$ is maximal, that means that each $\left|\Psi_{\sigma_{i}}^{\alpha}\right\rangle$ is entangled. So, the simplex associated with a maximal partition shows us the 'localisation of entanglement' for ensembles representing the state $\rho$. If there are several maximal partitions, each of them represents a class of ensembles which can represent $\rho$. Overlapping simplices indicate how similar are particular localisations of entanglement for different locally inequivalent ensembles representing the same state $\rho$. Now consider some special cases, beginning with trivial ones where separability polytopes degenerate.

\subsection{Trivial cases}

Begin with pure states. If $\rho$ is a pure state, then its separability polytope $\mathcal{K}(\rho)$ is a simplex.

Indeed, if $\rho$ is pure, then it is a projector on a pure state $|\Psi\rangle \in \mathcal{H}$. Let $\Sigma=\left\{\sigma_{1}, \ldots, \sigma_{M}\right\}$ is such a partition of $\mathfrak{A}$ that $|\Psi\rangle$ decomposes into a product

$$
|\Psi\rangle=\left|\Psi_{\sigma_{1}}\right\rangle \otimes \cdots \otimes\left|\Psi_{\sigma_{M}}\right\rangle
$$


such that each $\left|\Psi_{\sigma_{i}}\right\rangle$ is entangled. Then suppose that there is another partition $\Sigma^{\prime}=\left\{\sigma_{1}^{\prime}, \ldots, \sigma_{M}^{\prime}\right\}$ incomparable with with $\Sigma$ in the sense (曾). Then it turns out that $|\Psi\rangle$ has in addition to the decomposition (11) another one

$$
|\Psi\rangle=\left|\Psi_{\sigma_{1}^{\prime}}\right\rangle \otimes \cdots \otimes\left|\Psi_{\sigma_{M}^{\prime}}\right\rangle
$$

Then tracing out $|\Psi\rangle$ expressed as (11) with respect to a subsystem $\sigma_{i}$ which is not in $\Sigma^{\prime}$ (it must exist as $\Sigma, \Sigma^{\prime}$ are incomparable) we get a pure state. On the other hand, doing the same operation with $|\Psi\rangle$ expressed as (12) we get a mixed state. Therefore the conjectured $\Sigma^{\prime}$ does not exist, and we have the only partition $\Sigma$ for the state $\rho$.

The separability simplex for a pure state is obtained as follows. We take the simplex whose vertices are the subsystems of $\mathfrak{S}$ and collapse the vertices which belong to the same element of the partition $\Sigma$. In particular, when $\rho$ is fully entangled, the polytope collapses to a single point. This shows that the topological classification we provide is not complete with respect to LOCC (local operations + classical communications). It follows, for instance, from the existence of LOCC-inequivalent fully entangled tripartite mixed states [1, 7, 11].

However, for the special case of pure states of multi-qubit systems a more subtle classification exists based on the Carteret-Higuchi-Sudbery pure states decomposition [6]. This classification starts from the same simplex of subsystems but is more 'sparing' and does not collapse to the extent proposed here. For further details the reader is referred to [10].

The next trivial case is that of bipartite systems. It is degenerate for the proposed construction as the variety of entanglement patterns is quite poor, that is, a bipartite state can be either separable or entangled and there are no intermediate cases.

\subsection{Three qubits.}

Three-qubit systems provide us first non-trivial examples of essentially different entanglement patterns for mixed states. Although the number of subsystem is still small to provide 'interesting' polytopes. Referring to the classification [8] of mixed 3-qubit states. Let $\mathfrak{A}=\{A, B, C\}$, there are the following classes of states: 


\begin{tabular}{|c|c|}
\hline & $\begin{array}{l}\text { Fully entangled states for which the separability polytope } \\
\text { shrinks to a single point. An example of such state is, say } \\
\text { GHZ state [9]. }\end{array}$ \\
\hline & $\begin{array}{l}\text { 1-qubit biseparable states which can be separated as } \Sigma= \\
\{A, B C\} \text { or } \Sigma=\{B, A C\} \text { or } \Sigma=\{C, A B\} \text {. For them the } \\
\text { separability polytopes are } 1 \text {-simplices, that is, two points } \\
\text { connected by an edge (see section (1.3). An example of } \\
\text { such state may be a product state of a pure 1-qubit state } \\
\text { and fully entangled state of the remaining two qubits. }\end{array}$ \\
\hline & $\begin{array}{l}\text { 2-qubit biseparable states which can be separated as } \\
\Sigma=\{\{A, B C\},\{B, A C\}\} \text { and similar ones where } B, C \\
\text { are not separated out, respectively. The explicit examples } \\
\text { of such states can be found in [8]. For them the separa- } \\
\text { bility polytopes are disjoint unions of two 1-simplices. }\end{array}$ \\
\hline & $\begin{array}{l}\text { An interesting and slightly counterintuitive example } ₫ \\
\text { is that of } 3 \text {-biseparable states which are separable with } \\
\text { respect to any bipartite split. For them the separability } \\
\text { polytopes are disjoint unions of three 1-simplices. }\end{array}$ \\
\hline & $\begin{array}{l}\text { Finally, separable states provide us one more trivial exam- } \\
\text { ple - for them the separability polytopes are } 2 \text {-simplices } \\
\text { (that is, triangles). }\end{array}$ \\
\hline
\end{tabular}

Remark. I order to avoid confusion I emphasise that the lines drawn above show that the appropriate parties are separable rather than entangled since usually, when entanglement pictures are drawn, the lines denote entangling interactions.

\subsection{Physical meaning of separability polytopes}

Any mixed state $\rho$ of an ensemble can be represented as a mixture of pure states. For classical systems this representation is unique. In contrast, any mixed state of a quantum system can be represented as an ensemble of pure states in infinitely many inequivalent ways. When the system in question is composite, multipartite, for any given particular ensemble $\mathcal{J}$ representing 
the state $\rho$ we may ask: "is any of pure states forming $\mathcal{J}$ is separable or not with respect to this or that partition of the set of subsystems $\mathfrak{A}$ of the system S?' Separability polytopes are exactly the objects which capture this property. They show how many inequivalent (from the point of view of localisation) ensembles can form a given state $\rho$.

'Relatively local' transformations What can separability polytopes also serve for, is to broaden the group of unitary transformations with respect to which the entanglement picture of a multipartite state remains unchanged. We can derive it using the fact that separability polytopes are not given ad hoc arbitrary ones. Their maximal simplices are associated with partitions. Recall that the collection of all possible partitions of a set $\mathfrak{A}$ is partially ordered by the relation (1). Moreover, the set of partitions has the structure of a lattice [5], that is, for any two partitions there exist their least upper bound and the greatest lower bound with respect to relation (11). This means, in turn, that we have a well defined operations of JOIN $\vee$ and MEET $\wedge$ on simplices:

$$
\begin{aligned}
& \mathcal{S}_{1} \vee \mathcal{S}_{2}=\sup \left\{\mathcal{S}_{1}, \mathcal{S}_{2}\right\} \\
& \mathcal{S}_{1} \wedge \mathcal{S}_{2}=\inf \left\{\mathcal{S}_{1}, \mathcal{S}_{2}\right\}
\end{aligned}
$$

In the sequel we shall be interested only in $\vee$ operation. Let $\mathcal{S}=\sup \left\{\mathcal{S}_{i}\right\}$ and consider the class (a group, in fact) $\mathbf{U}_{\rho}$ of unitary transformation which are local with respect to the partition $\mathcal{S}$ which is the refinement of all maximal simplices (=partitions) of the separability polytope $\mathcal{K}(\rho)$. Then any transformation from this group applied to $\rho$ keeps its separability polytope $\mathcal{K}(\rho)$ unchanged, however $\mathbf{U}_{\rho}$ is in general greater than the group of 'genuinely' local transformations.

\section{Implementations of quantum gates from topo- logical perspective}

In this section I consider a register $\mathfrak{S}$ of $N$, say, qubits (the number of

individual degrees of freedom is not important) and stepwise implementation 
of an arbitrary unitary transformation on it. By 'stepwise' I mean that at each time step only a 2-qubit transformation is carried out. Proceeding this way, one can implement, having a very limited scope of resources in disposal, an arbitrary unitary transformation on the whole system $\mathfrak{S}$ up to any given accuracy [2].

Now suppose we have a compound system $\mathfrak{S}$. In the sequel call it register, and call its subsystems qubits sequel. Let us see what happens with the separability polytope of the state of the register in course of the stepwise execution of this simulation. We start with a certain initial (in general, mixed) state $\rho_{0}$ with the separability polytope $\mathcal{K}\left(\rho_{0}\right)$. As it was claimed in section 2.4, $\mathcal{K}\left(\rho_{0}\right)$ represents the equivalence classes of ensembles which can realise the state $\rho_{0}$. At first step, when we pass from $\rho_{0}$ to $\rho_{1}=\mathbf{U}_{0} \rho_{0} \mathbf{U}_{0}^{\dagger}$ we may view it as follows: pick an ensemble $\mathcal{J}$ of pure states associated with a particular maximal simplex of $\mathcal{K}\left(\rho_{0}\right)$ and apply $\mathbf{U}_{0}$ to each pure state forming $\mathcal{J}$.

For general $\mathbf{U}_{0}$ nothing certain can be said, but recall that we consider a special kind of transformations, namely, entangling at most two qubits. Let $\mathcal{J}$ be an ensemble representing $\rho$, associate with it a partition $\Sigma$ of $\mathfrak{S}$. The action of $\mathbf{U}_{0}$ may be either (i) entangling two qubits within one element of the covering associated with the a partition or (ii) 'breaking the border', that is, entangling two qubits from different elements of the partition. Both these options have direct topological interpretation.

In case (i) the appropriate partition remains intact, in case (ii) two elements of the partition merge. In terms of simplices option (ii) means that an edge of appropriate simplex collapses to a vertex. In both cases this transition is a simplicial mapping (see section 1.3). To see the real action of $\mathbf{U}_{0}$ we must apply itto all non-equivalent ensembles (= maximal simplices of $\left.\mathcal{K}\left(\rho_{0}\right)\right)$. As a result, the whole complex undergoes a simplicial contracting some edges of $\mathcal{K}\left(\rho_{0}\right)$ may collapse to vertices.

Now recall that vertices of $\mathcal{K}\left(\rho_{0}\right)$ are subsets of the set $\mathfrak{A}$ of subsystems of $\mathfrak{S}$. So, it may happen that the same subset will be obtained as different junctions. For example, suppose we have the following edges (= pairs of disjoint subsets)

$$
e_{1}=\{\{1,2\},\{3\}\}, \quad e_{2}=\{\{1\},\{2,3\}\}
$$

and we see that they have no common vertices. When they both collapse, we get 


$$
\mathbf{U}_{0}\left(e_{1}\right)=\{1,2,3\}, \quad \mathbf{U}_{0}\left(e_{2}\right)=\{1,2,3\}
$$

which means that in the resulting complex the two vertices $\mathbf{U}_{0}\left(e_{1}\right), \mathbf{U}_{0}\left(e_{2}\right)$ should be identified. And again, this operation never 'breaks edges' that is why it is a simplicial mapping.

So, any two-qubit unitary transform on a composite system is associated with a simplicial mapping of appropriate separability polytopes. But, as it was mentioned above, any unitary transformation can be implemented as a sequence (in general infinite) of 2-qubit transformations.

Now recall that we are dealing with finite-dimensional systems for which any set of states sharing the same entanglement pattern is of non-zero measure. That means that we can arrange the implementation in such a way that after a finite number of steps the separability polytope of the state will not change any longer. As a result, taking into account that compositions of simplicial mappings are always simplicial, we can conclude that

Any unitary transformation of any given state of a finite-dimensional multipartite system can be associated with a simplicial mapping of their separability polytopes.

Remark. Note that if we consider a unitary transformation per se, not implemented as a sequence of at most 2-qubit operations, it has in general nothing to do with simplicial mappings. Therefore a particular simplicial mapping is associated with the realisation of the transformation rather than with the transformation itself.

\section{Concluding remarks}

I have introduced an essentially non-numerical characterisation of mixed states of multipartite quantum systems. With each state $\rho$ a polytope purely topological object which can be realised as a polyhedron in a Euclidean space - is associated. These polytopes capture the localisation properties of pure states forming ensembles which can represent the state $\rho$. (by localisation of a pure state I mean with respect to which groups of subsystems it is entangled). 
It turns out that the presented characterisation is related with the simulation of arbitrary unitary operations by 1- and 2-qubit gates. Namely, to each particular implementation of such kind a simplicial mapping - well known construction in algebraic topology - can be put into correspondence.

Acknowledgments. I am grateful to the members of Quantum Information Group, ISI for permanent attention to the work and many helpful comments and discussions. Valuable comments from Demosthenes Ellinas, Markus Grassl and Philippe Jorrand are appreciated. The work was carried out under the auspices of the EU grant Q-ACTA 'Quantum computation: novel algorithms and their many-body implementations' (IST-1999-10596).

\section{References}

[1] A. Acín, E. Jane, W. Dür, and G. Vidal, Optimal distillation of a GHZ state, Phys. Rev. Lett., 85:4811, 2000; eprint quant-ph/0007042

[2] A. Barenco , C. H. Bennett, R. Cleve, D. P. DiVincenzo , N. Margolus , P. Shor , T. Sleator , J. Smolin , H. Weinfurter, Elementary gates for quantum computation, Phys.Rev. A, 52:3457, 1995

[3] C.H. Bennett, G. Brassard, C. Crépeau, R. Jozsa, A. Peres, and W.K. Wootters, Phys. Rev. Lett. 70: 1895 (1993)

[4] C.H. Bennett, D.P. DiVincenzo, T. Mor, P.W. Shor, J.A. Smolin, B.M. Terhal, Unextendible Product Bases and Bound Entanglement, Phys.Rev.Lett. 82:5385, 1999

[5] G. Birkhoff, Lattice theory, Providence, Rhode Island , 1967.

[6] H. A. Carteret, A. Higuchi, A. Sudbery, Multipartite generalisation of the Schmidt decomposition, J. Math. Phys. 41, 7932, 2000

[7] W. Dür, G. Vidal, and J. I. Cirac, Three qubits can be entangled in two inequivalent ways, Phys. Rev. A, 62:062314, 2000; eprint quantph/0005115 
[8] W. Dür, J. I. Cirac Classification of multi-qubit mixed states: separability and distillability properties, Phys. Rev. A 61:042314, 2000; eprint quant-ph/9911044

[9] D.M.Greenbberger, M.Hoorne, A.Zeilinger, Bell's theorem, quantum theory, and conceptions of the Universe, M.Kafatos (ed.), Kluwer, Dordrecht (1989)

[10] I. Raptis and R. R. Zapatrin, Decomposition of pure states of a quantum register, Quantum Computers and Computation, to appear in 2002; eprint quant-ph/0010104.

[11] F. Verstraete, J. Dehaene, and B. De Moor, The Lorentz singular value decomposition and its applications to pure states of 3 qubits, Phys. Rev. A, 65:032308, 2002; eprint quant-ph/0108043 